\title{
Effect of EDTA on the Growth Kinetics and Structural and Optical Properties of KDP Crystal
}

\author{
Anisur Rahman ${ }^{1}$ and Jiban Podder ${ }^{2}$ \\ ${ }^{1}$ Interdepartment of Physics, The University of Asia Pacific, Dhaka 1209, Bangladesh \\ ${ }^{2}$ Department of Physics, Bangladesh University of Engineering and Technology, Dhaka 1000, Bangladesh
}

Correspondence should be addressed to Jiban Podder, jpodder@phy.buet.ac.bd

Received 6 September 2010; Accepted 13 December 2010

Academic Editor: Sang-Kook Han

Copyright (c) 2010 A. Rahman and J. Podder. This is an open access article distributed under the Creative Commons Attribution License, which permits unrestricted use, distribution, and reproduction in any medium, provided the original work is properly cited.

\begin{abstract}
The solubility of potassium dihydrogen phosphate (KDP) was determined for five different temperatures. Metastable zone width of aqueous solutions of KDP saturated at different temperatures was determined by nucleation method in pure form and adding a well-known chelating agent ethylenediaminetetraacetic acid $(0.5 \mathrm{~mol} \%$ EDTA). The induction period $\tau$ was measured, experiments were performed at selected degrees of supersaturation $\left(\mathrm{C} / \mathrm{C}^{*}\right)$, and the critical nucleation parameters like interfacial energy $(\sigma)$ were calculated, based on the classical theory of nucleation. The structural and optical properties of the grown crystals have been investigated. FTIR analysis has been carried out to characterize the grown crystals. Band gap has been calculated from transmission spectra.
\end{abstract}

\section{Introduction}

Recently potassium dihydrogen phosphate (KDP) crystals have created considerable interest among researchers because of its Piezo-electric, nonlinear optical, and electro-optical properties, and also it is widely used in X-ray monochromators [1]. So attention is given to grow crystals from solutions with faster rates by adopting faster cooling rates, but faster cooling rates depend on the metastable zone width which is an essential parameter for the growth of large size crystals from solution since it is the direct measure of the stability of the solution in its supersaturated region. The larger the zone width the larger the stability [2]. Solutions with narrow metastable zone width very often end up with secondary nucleation. Srinivasan et al. introduced a novel method to enhance the metastable zone width for crystal growth from solutions by adding the chelating agent EDTA [3].

The deciding factor of the metastable zone width is the presence of the impurities. Most of the commercially available chemicals have impurities of heavy metals like $\mathrm{Cr}^{3+}, \mathrm{Fe}^{3+}$, and $\mathrm{Al}^{3+}$. These impurities induce the secondary nucleation and reduce the metastable zone width. Such impurities, having a long residence time in the adsorbed layer, become stoppers for the elementary steps and at higher supersaturations enhance the surface heterogeneous nucleation rate. The distribution of metals becomes more difficult by the addition of EDTA, as it usually binds to a metal cation through two amines and four carboxylates. Basically, the molecular size of EDTA is far higher than KDP, apart from which the fact that it makes complexes with heavy metals, further increases in size, hence there is no chance for EDTA molecule complexes to penetrate into KDP molecular structure [4]. Thus, it is possible to use EDTA for increasing the metastable zone width without the danger of EDTA getting into KDP molecular structure.

At room temperature, KDP has a noncentrosymmetric tetragonal lattice. The unit cell dimensions are $a=b=$ $7.434 \AA$ and $c=6.945 \AA$. KDP has a phase transition to ferroelectric from its paraelectric state at $123 \mathrm{~K}$. The KDP lattice is composed of two sets of $\mathrm{PO}_{4}$ groups linked to each other by hydrogen bonds. With the aim of improving the quality of KDP crystals with better optical properties, an attempt has been made in the present work to grow the KDP crystals by doping it with EDTA (concentration of $0.5 \mathrm{~mol} \%$ ) and to study the effect on the nucleation parameters and structural and optical properties. 


\section{Experiment}

2.1. Solubility Studies. In the present work, the solubility of KDP is determined for five different temperatures, namely, $30,35,40,45$, and $50^{\circ} \mathrm{C}$. Solubility studies were carried out in a constant temperature water bath (CTB) with cryostat facility with an accuracy of $\pm 0.01 \mathrm{~K}$.

2.2. Determination of Metastable Zone Width. The metastable zone width studies of pure and doped KDP solutions were carried out by adopting the polythermal method [5]. The KDP solution $(200 \mathrm{~mL})$ saturated at $30^{\circ} \mathrm{C}$ was prepared according to the solubility diagram with continuous stirring using magnetic stirrer. Then the solution was filtered by the filtration pump and Whatman filter paper of pore size $11 \mu \mathrm{m}$. Two similar beakers with $100 \mathrm{~mL}$ solution each were used, the first beaker contains pure KDP solution whereas the second beaker contains $0.5 \mathrm{~mol} \%$ EDTA-doped KDP solutions, respectively. Then pure and doped KDP solutions were kept in a CTB with cryostat facility. The solutions were stirred continuously for a period of 6 hours for stabilization using magnetic stirrer. It was slowly cooled at a desired cooling rate of $4 \mathrm{~K} / \mathrm{h}$; until the first crystal appeared, the temperature was instantly recorded. The difference between the saturation temperature and nucleation temperature was taken to be the maximum undercooling $\Delta t_{\max }$. This gives the metastable zone width of the solution. This experiment is repeated for different saturation temperatures $35,40,45$, and $50^{\circ} \mathrm{C}$, and the corresponding metastable zone widths were measured.

2.3. Determination of Induction Period. The induction period " $\tau$ " is a measure of the "ability" of the solution to remain supersaturated and is determined experimentally for KDP solutions with and without the presence of EDTA by adopting isothermal method [6]. The supersaturated solutions were prepared to conduct induction time experiments. The required level of supersaturation was achieved by dissolving the required amount of KDP (with and without EDTA) in the solvent, which is above the saturation concentration. The mixture is then heated a few degrees above the saturation temperature $\left(30^{\circ} \mathrm{C}\right)$ to ensure that all the material is dissolved. The solution is then cooled to the saturation temperature $\left(30^{\circ} \mathrm{C}\right)$ where the solution becomes supersaturated to the required degree of supersaturation. Once the nucleation occurred, it grew quickly and a bright sparkling particle was seen. The time of observation of the sparkling particle in the cell from the time at which the solution reaches the saturation temperature gives the induction period of nucleation. Experiments were performed at selected degrees of supersaturation $\left(C / C^{*}\right)$, namely, $1.07,1.1,1.13$, and 1.16, ( $C$ being the concentration of solute in supersaturated solution and $C^{*}$ is the saturated concentration).

\section{Nucleation Kinetics of KDP}

The interfacial energy $\sigma$ of the interface between the growing crystal and the surrounding mother phase plays an important role in the nucleation of crystals. This complex parameter has been determined by conducting nucleation experiments using the saturated solution. The relationship between rate of nucleation (representing the number of nuclei formed per unit time per volume) and induction period can be expressed as [7]

$$
\begin{aligned}
& J=\frac{1}{\tau}=A \cdot \exp \left(-\frac{\Delta G^{*}}{k T}\right) \\
& =A \exp \left(-\frac{16 \pi \sigma^{3} v^{2}}{3 k^{3} T^{3}(\ln S)^{2}}\right), \\
& \ln (\tau)=-\ln (A)+\frac{16 \pi \sigma^{3} v^{2}}{3 k^{3} T^{3}(\ln S)^{2}},
\end{aligned}
$$

$\tau$ is the induction period of the solution at temperature $T$, $v$ is the molar crystal volume, $k$ is the Boltzman constant, and $A$ is constant. $S$ is the supersaturation ratio $\left(S=C / C^{*}\right)$. The function $\ln A$ weakly depends on temperature, and hence there is a linear dependence between $\ln \tau$ and $1 /(\ln S)^{2}$ at constant temperature (Figure 2). A plot of $\ln \tau$ against $1 /(\ln S)^{2}$ is a straight line. The intercept of the straight line on the $y$-axis gives the value of $\ln A$. Equation (3) suggests a straight line fit for $\ln \tau$ against $1 /(\ln S)^{2}$ with a slope $m$ given by [5]

$$
m=\frac{16 \pi \sigma^{3} V^{2} N_{A}}{3 R^{3} T^{3}} .
$$

In some studies [8], linear relationship between $1 /(\ln S)^{2}$ and $\ln (\tau)$ is not represented by one line, but two different straight lines can be drawn, one of which represents homogeneous nucleation and the other represents heterogeneous nucleation. The interfacial energy $\sigma$ of the solid relative to the solution has been calculated from the slope of the line as

$$
\sigma^{3}=\frac{3 R^{3} T^{3} m}{16 \pi V^{2} N_{A}},
$$

where $V$ is the specific volume and is obtained by $V=$ Molecular Weight/Density $\times N_{A}, R$ is the gas constant, and $N_{A}$ is Avogadro's number.

\section{Characterization}

4.1. FTIR Analysis. FTIR spectroscopy is effectively used to identify the functional groups in order to determine the molecular structure of the compounds. The FTIR spectrum was recorded by $\mathrm{KBr}$ pellet technique in the range $400-4000 \mathrm{~cm}^{-1}$. Figure 4 shows the FTIR spectra of the pure and EDTA-added KDP crystal. There is a broad envelope between $3700 \mathrm{~cm}^{-1}$ and $2500 \mathrm{~cm}^{-1}$. It includes $\mathrm{O}-$ $\mathrm{H}$ stretching vibrations of KDP. Hydrogen bonding within the crystal is suggested to be the cause for the broadening of the peak [9]. The presence of water is well supported by its bending vibrations occurring at the band $1630 \mathrm{~cm}^{-1}[10]$. The peaks below $1200 \mathrm{~cm}^{-1}$ are due to $\mathrm{PO}_{4}$ vibrations. The broad envelopes observed between 2300 and $3600 \mathrm{~cm}^{-1}$ are mainly due to $\mathrm{P}-\mathrm{OH}$ stretching of $\mathrm{H}_{2} \mathrm{PO}_{4}$. Intense absorption observed at $536 \cdot 4 \mathrm{~cm}^{-1}, 435 \cdot 9 \mathrm{~cm}^{-1}$, and $413 \cdot 7 \mathrm{~cm}^{-1}$ is due to $\mathrm{P}-\mathrm{OH}$ deformation. 


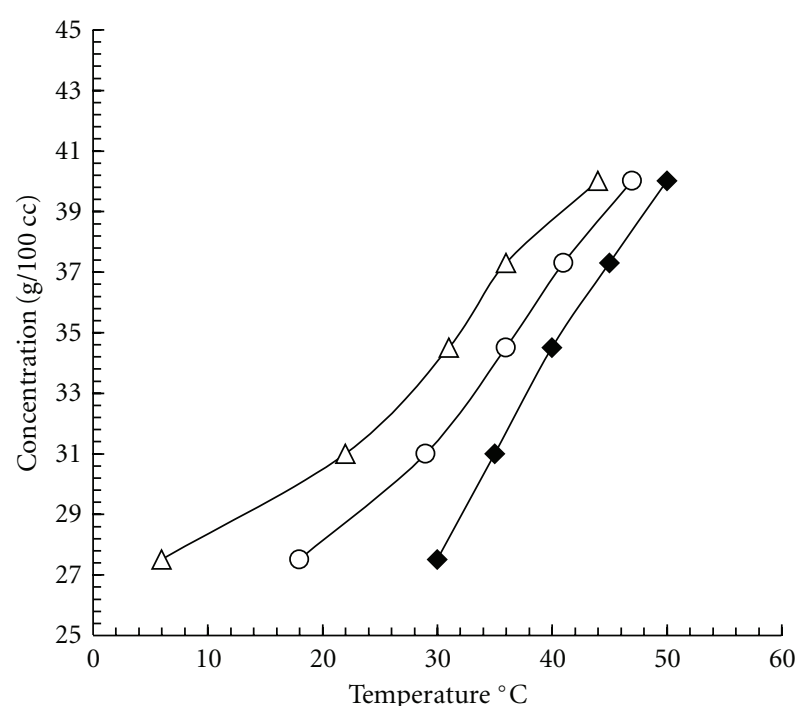

Solubility curve of pure KDP

$-\mathrm{O}-$ Nucleation curve of pure KDP

$-\triangle$ Nucleation curve of KDP +0.5 mol \% EDTA

FIgURE 1: Solubility and metastable zone width for different saturation temperatures for pure and EDTA-added KDP solution.

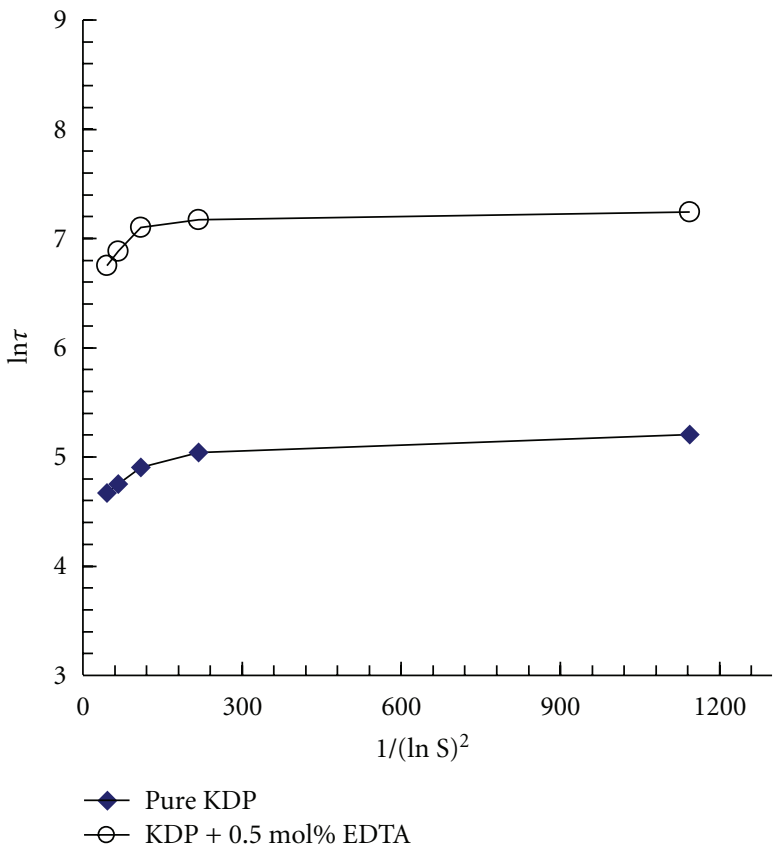

Figure 2: $\ln \tau$ versus $1 /(\ln S)^{2}$ of KDP and EDTA-added KDP solution.

4.2. Energy Band Gap Measurement. For optical applications, the crystal should be highly transparent in the considerable region of wavelength $[11,12]$. The good transmission of the crystal in the entire visible region suggests its suitability for second harmonic generation devices shown in Figure 5 [13]. The dependence of the optical absorption coefficient with the photon energy helps to study the band structure and

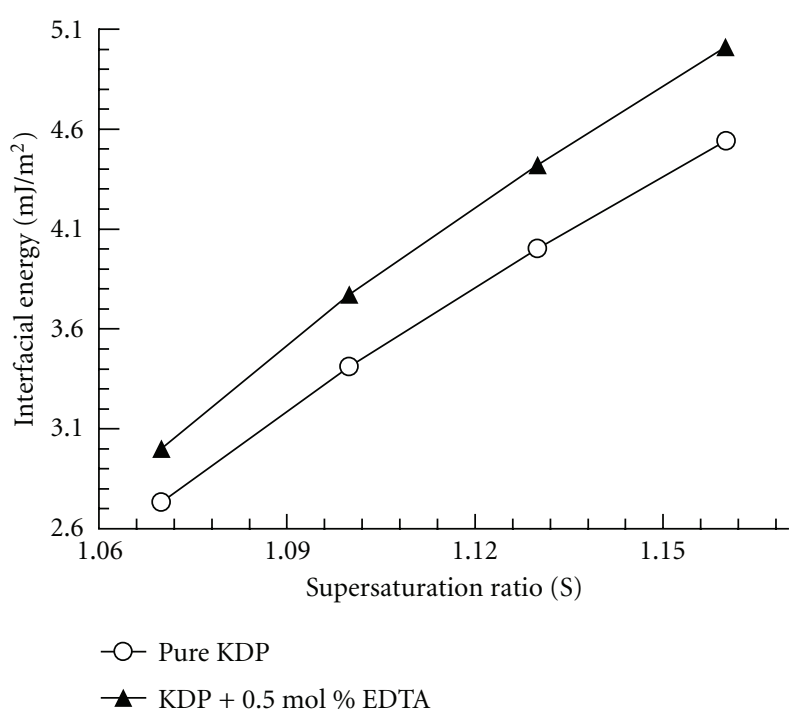

FIGURE 3: Interfacial energy versus supersaturation ratio of pure and EDTA-added KDP solution.

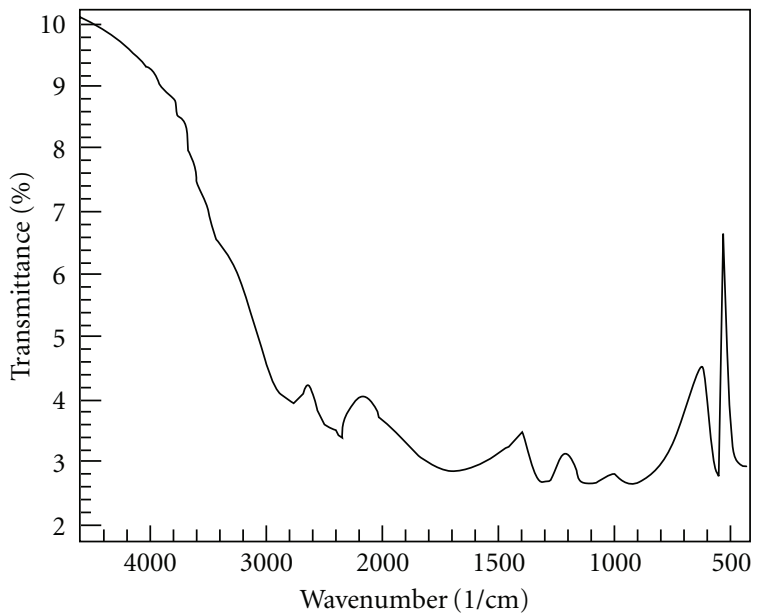

(a) Pure KDP

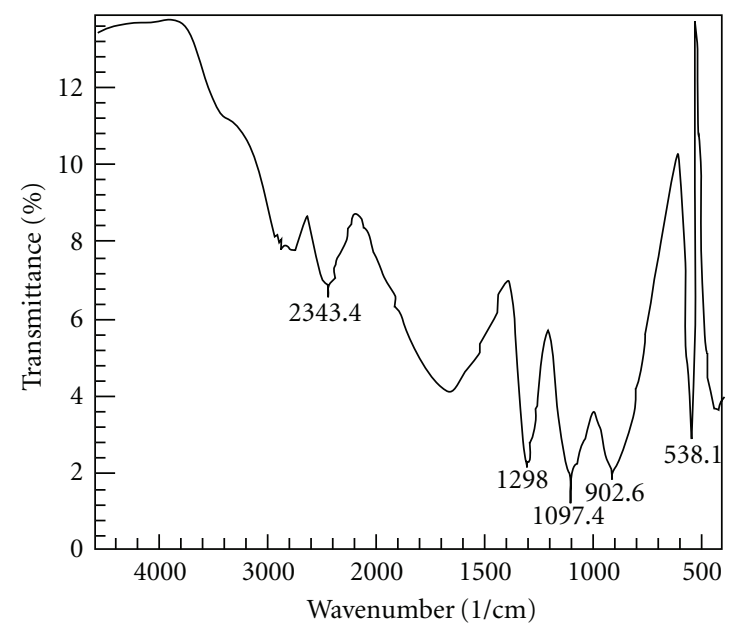

(b) $\mathrm{KDP}+0.5 \mathrm{~mol} \% \mathrm{EDTA}$

FIGURE 4: FTIR spectrum for (a) pure KDP and (b) EDTA added KDP crystal. 


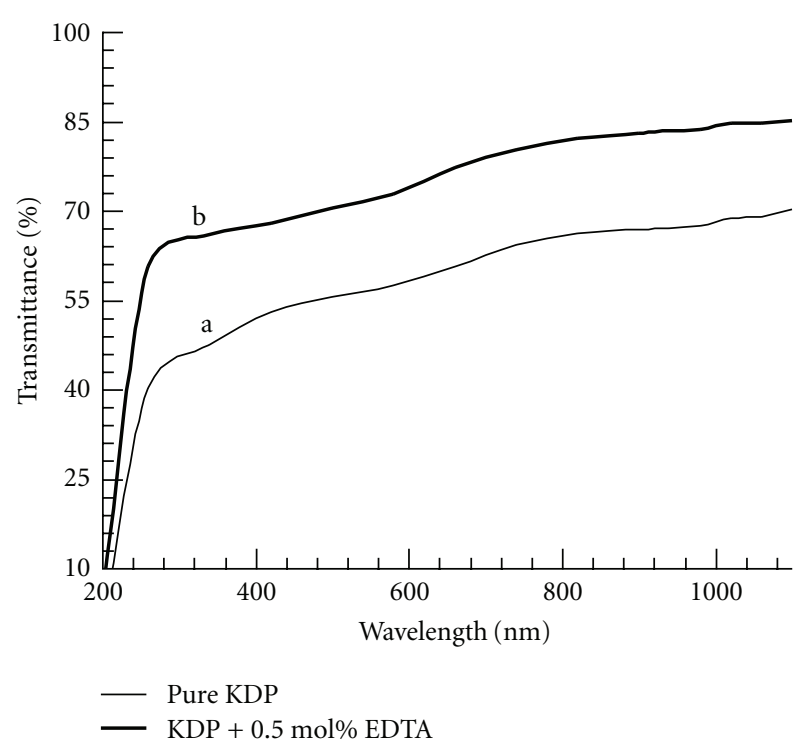

FIgURe 5: UV-VIS spectra for (a) pure KDP and (b) EDTA added KDP crystal.

TABLE 1

\begin{tabular}{lc}
\hline Material & Optical band gap $(\mathrm{eV})$ \\
\hline Pure KDP & 1.38 \\
KDP +0.5 mol\% EDTA & 1.55 \\
\hline
\end{tabular}

the type of transition of electrons. The optical absorption coefficient $(\alpha)$ was calculated from the transmittance using the following relation [14]:

$$
\alpha=\frac{1}{d} \log \left(\frac{1}{T}\right)
$$

where $T$ is the transmittance and $d$ is the thickness of the crystal. For direct transition, the absorption coefficient is given by

$$
\alpha_{d}=\frac{A\left(h \nu-E_{g}\right)^{r}}{h \nu}
$$

where $A$ is constant involving the properties of the bands, $h \nu=$ photon energy, $E_{g}=$ Energy gap, $r=1 / 2$ for allowed transition, and $r=3 / 2$ for forbidden transition. A plot of $h \nu$ versus $(\alpha h \nu)^{2}$ would give the value of optical band gap $E_{g}$. The value of band gap has been calculated by taking the intercept on $x$-axis of Figure 6.

4.3. Results and Discussion. The grown crystals of pure KDP and EDTA-added KDP are shown in Figure 7. It is observed from Figure 1 that the metastable zone width is enhanced due to the addition of EDTA but the zone width decreases with the increase in temperature. Also it has been observed from the experiment that the addition of EDTA reduces the number of the secondary nucleation in the solution medium.

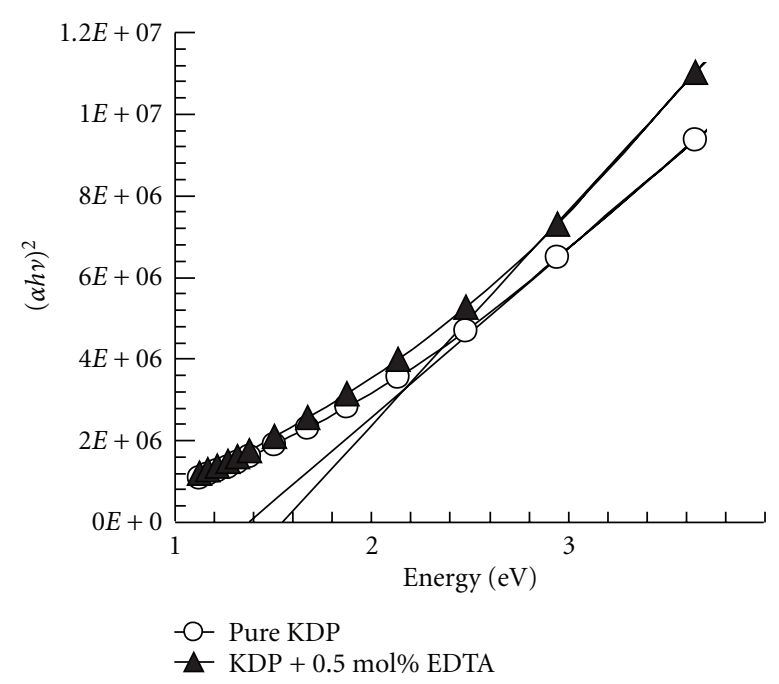

FIgURE 6: Plot of $(\alpha h \nu)^{2}$ versus photon energy for pure and EDTAadded KDP crystal.

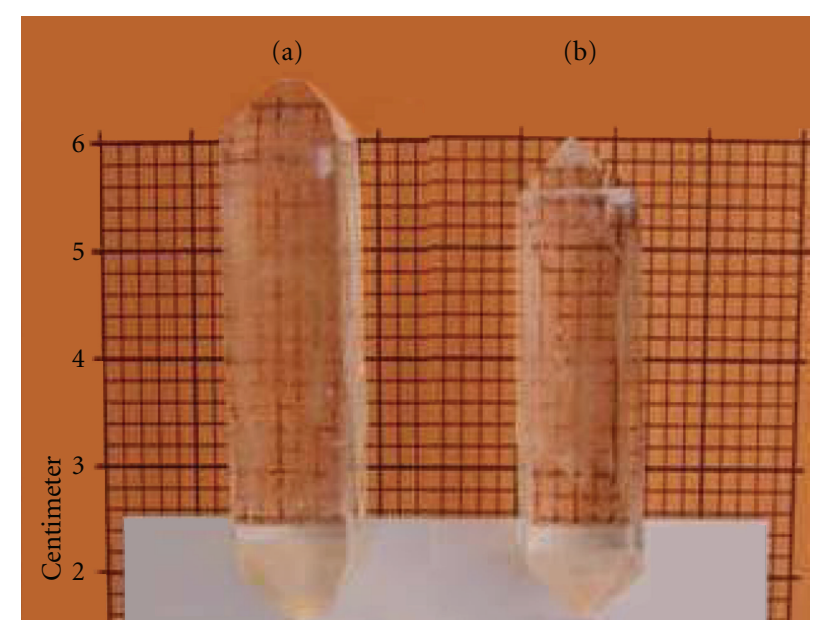

Figure 7: Photograph of crystals grown by slow evaporation method: (a) pure KDP and (b) KDP with 0.5 mol\% EDTA.

From Figure 3, it is observed that the interfacial energy is increasing with the addition of EDTA. Thus the probability of nucleation is decreasing considerably as the interfacial energy is having a positive term and acts to destabilize the nucleus and this is why, secondary nucleation was observed to decrease.

By comparing the FTIR spectra of doped and undoped crystals, it can be easily concluded that there was no incorporation of EDTA in the KDP molecular structure. From optical transmission spectra it is seen that the addition of EDTA has increased the optical transparency as well as band gap of the crystal from 1.38 to $1.55 \mathrm{eV}$ (Table 1). Since the material has good optical transmission characteristics in the wavelengths range $280-1090 \mathrm{~nm}$, the material is suitable for generating second harmonics in the blue-green region [15]. 


\section{Conclusions}

Pure and EDTA-added KDP crystals were grown by slow evaporation technique. The solubility data was found to satisfy a linear relation. It has also been found that EDTA addition enhances the metastable zone width significantly compared to the pure solution. This may be due to the suppression of chemical activity of the metal ions in the KDP solution. From the study of induction period, the critical nucleation parameters are calculated, and it is observed that the values of $\tau$ and $\sigma$ increase with the addition of EDTA. The FTIR spectral analysis reveals the presence of functional groups and bonds of the material. From FTIR analysis it can be inferred that EDTA can be used effectively as an additive to increase the optical property of the crystal because EDTA cannot enter into KDP molecular structure. The UVVIS spectra analysis reveals that the transmission efficiency improves considerably for addition of EDTA. This study may prove to be helpful to obtain high-quality single crystals for optoelectronic applications as well as to apply faster growth rates of KDP crystals.

\section{References}

[1] N. P. Rajesh, V. Kannan, P. Santhana Raghavan, P. Ramasamy, and C. W. Lan, "Nucleation studies and crystal growth of $\left(\mathrm{NH}_{4}\right) \mathrm{H}_{2} \mathrm{PO}_{4}$ doped with thiourea in supersaturated aqueous solutions," Materials Chemistry and Physics, vol. 76, no. 2, pp. 181-186, 2002.

[2] H. E. Buckley, Ed., Crystal Growth, John Wiley \& Sons, New York, NY, USA, 1951.

[3] K. Srinivasan, K. Meera, and P. Ramasamy, "Enhancement of metastable zone width for solution growth of potassium acid phthalate," Journal of Crystal Growth, vol. 205, no. 3, pp. 457459, 1999.

[4] N. P. Rajesh, K. Meera, K. Srinivasan, P. Santhana Raghavan, and P. Ramasamy, "Effect of EDTA on the metastable zone width of ADP," Journal of Crystal Growth, vol. 213, no. 3-4, pp. 389-394, 2000.

[5] R. Sankar, C. M. Raghavan, and R. Jayavel, "Nucleation kinetics and growth aspects of semi organic non-linear optical bis thiourea cadmium acetate single crystals," Crystal Research and Technology, vol. 41, no. 9, pp. 919-924, 2006.

[6] N. P. Zaitseva, L. N. Rashkovich, and S. V. Bogatyreva, "Stability of $\mathrm{KH}_{2} \mathrm{PO}_{4}$ and $\mathrm{K}(\mathrm{H}, \mathrm{D})_{2} \mathrm{PO}_{4}$ solutions at fast crystal growth rates," Journal of Crystal Growth, vol. 148, no. 3, pp. 276-282, 1995.

[7] O. Sahin, H. Dolas, and H. Demir, "Determination of nucleation kinetics of potassium tetraborate tetrahydrate," Crystal Research and Technology, vol. 42, no. 8, pp. 766-772, 2007.

[8] T. Kanagasekaran, M. Gunasekaran, P. Srinivasan, D. Jayaraman, R. Gopalakrishnan, and P. Ramasamy, "Studies on growth, induction period, interfacial energy and metastable zonewidth of m-nitroaniline," Crystal Research and Technology, vol. 40, no. 12, pp. 1128-1133, 2005.

[9] P. V. Dhanaraj, N. P. Rajesh, P. Ramasamy, M. Jeyaprakasan, C. K. Mahadevan, and G. Bhagavannarayana, "Enhancement of stability of growth, structural and NLO properties of KDP crystals due to additive along with seed rotation," Crystal Research and Technology, vol. 44, no. 1, pp. 54-60, 2009.
[10] C. N. Banwell and E. M. McCash, Fundamentals of Molecular Spectroscopy, McGraw-Hill, New York, NY, USA, 4th edition, 1994.

[11] V. Krishnakumar and R. Nagalakshmi, "Crystal growth and vibrational spectroscopic studies of the semiorganic nonlinear optical crystal-bisthiourea zinc chloride," Spectrochimica Acta Part A, vol. 61, no. 3, pp. 499-507, 2005.

[12] V. Krishnakumar and R. J. Xavier, "Vibrational analysis of 1,4-diaminoanthraquinone and 1,5-dichloroanthraquinone: a joint FTIR, FT-Raman and scaled quantum mechanical study," Spectrochimica Acta Part A, vol. 61, no. 8, pp. 17991809, 2005.

[13] V. Venkataramanan, S. Maheswaran, J. N. Sherwood, and H. L. Bhat, "Crystal growth and physical characterization of the semiorganic bis(thiourea) cadmium chloride," Journal of Crystal Growth, vol. 179, no. 3-4, pp. 605-610, 1997.

[14] S. Krishnan, C. J. Raj, S. Dinakaran, and S. J. Das, "Investigation of optical band gap in potassium acid phthalate single crystal," Crystal Research and Technology, vol. 43, no. 6, pp. 670-673, 2008.

[15] K. Nagarajan, S. Kumara Raman, and R. Sundaram, "Growth and characterization of thiourea mixed cadmium-lead chloride-a nonlinear optical crystal," Crystal Research and Technology, vol. 44, no. 4, pp. 421-424, 2009. 

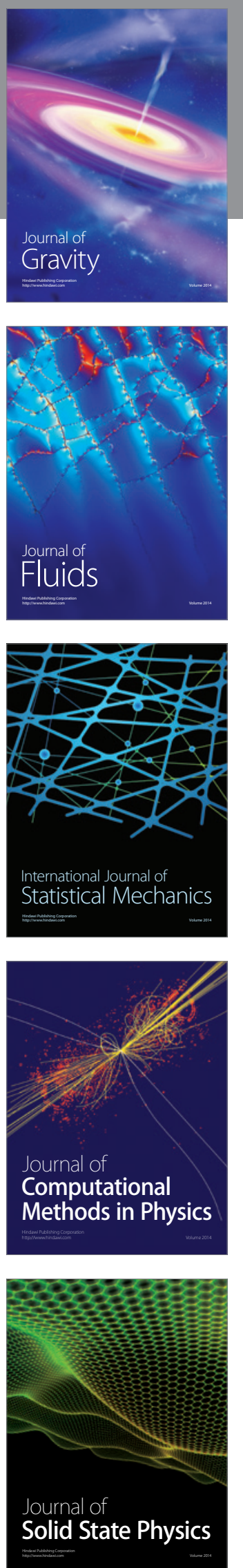

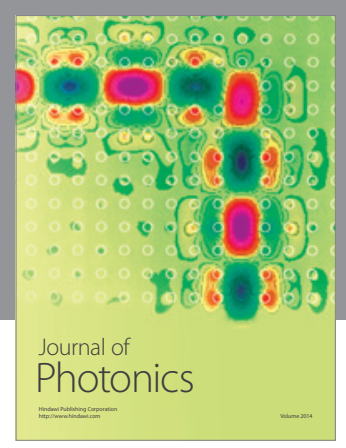

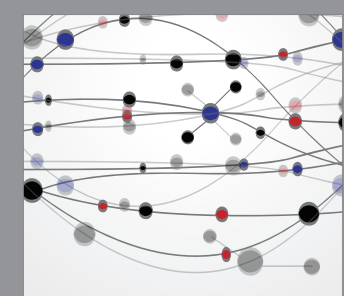

The Scientific World Journal
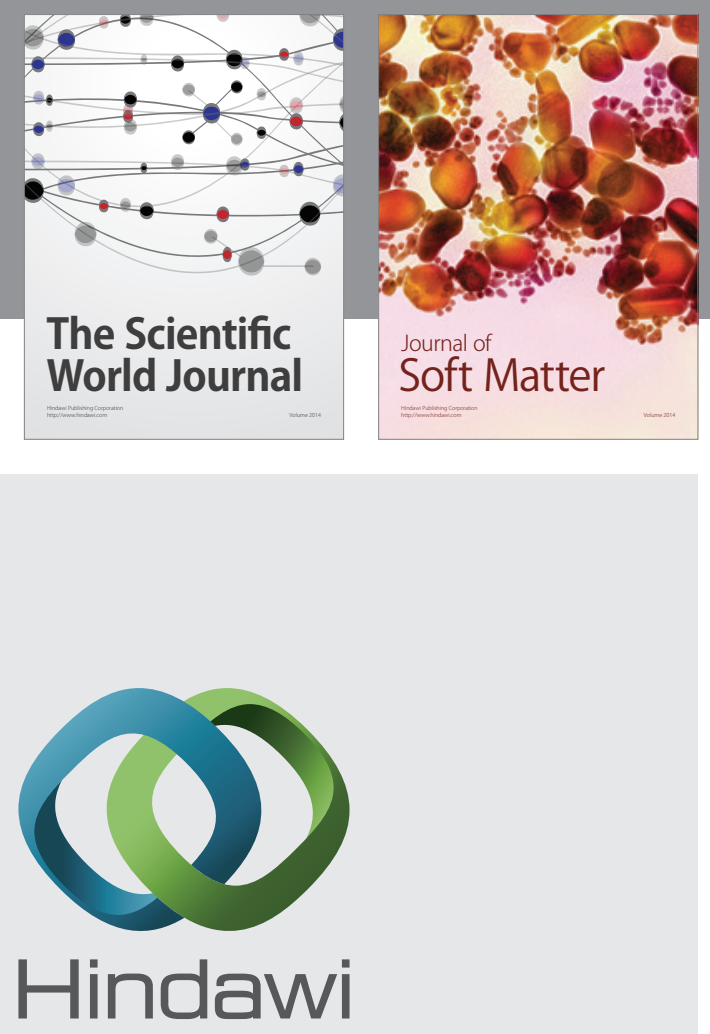

Submit your manuscripts at

http://www.hindawi.com
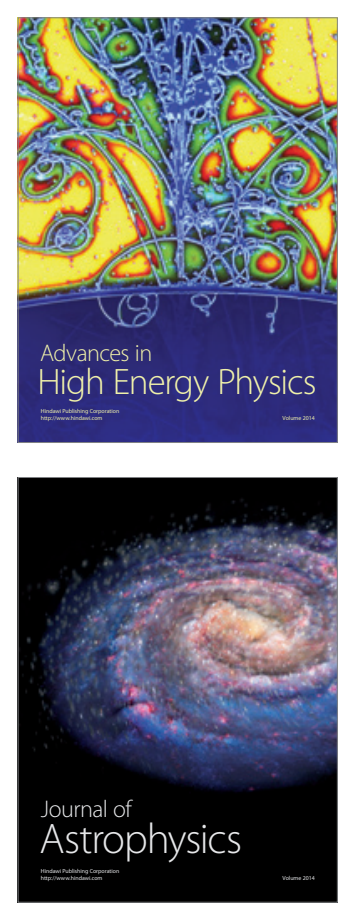
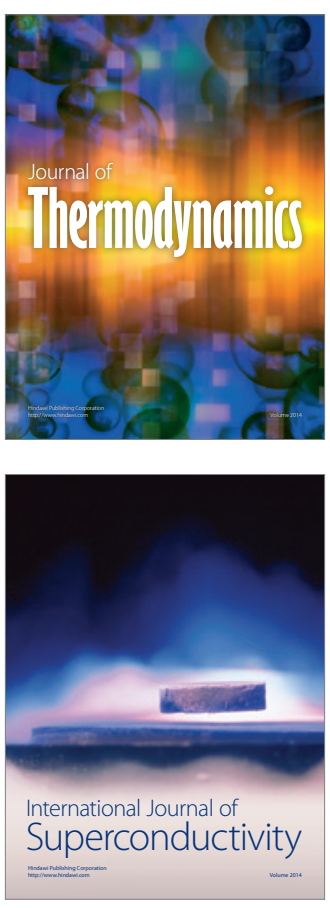
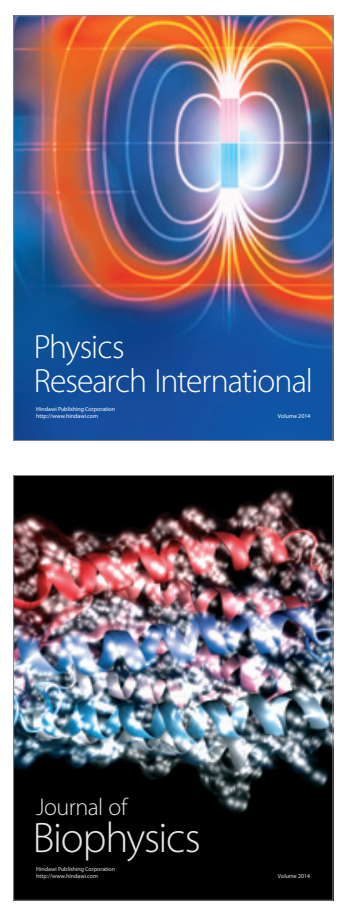
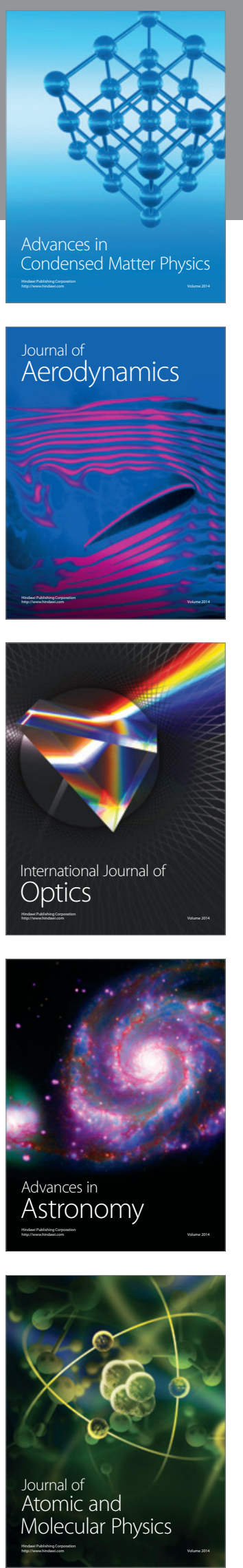